\title{
Glactomannan Index as a Rapid Diagnostic Tool for Invasive Pulmonary Aspergillosis in Patients with Chronic Lung Diseases
}

\author{
Asmaa Nasr Eldin Thabet ${ }^{1}$ and Kamal Abdel Sattar Ata $^{2}$ \\ ${ }^{1}$ Department of Microbiology and Immunology, ${ }^{2}$ Department of Chest Diseases, \\ Faculty of Medicine, Sohag University, Egypt \\ *Corresponding author
}

Keywords

Invasive aspergillosis,

Galactomannan antigen, Invasive fungal infections

\section{Article Info}

Accepted: 10 May 2019 Available Online: 10 June 2019
A B S T R A C T

Invasive aspergillosis has increased considerably in recent years; this serious infection was primarily described in severely immunocompromised patients and patients with moderate levels of immune suppression. We conducted this study on 85 patients admitted to the chest department and chest ICU with severe exacerbations of chronic lung diseases in the period from April 2018 to April 2019. We collected tracheal aspirates BAL fluid and blood samples for isolation of Aspergillus spp. by convectional culture and assay of galactomannan level in these samples using ELISA. Aspergillus spp. was isolated from $(14.3 \%)$ of tracheobroncheal aspirate samples, (11\%) BAL samples and $(2.4 \%)$ blood samples. Aspergillus fumigatus represented (70\%) of isolates while Aspergillus niger represented $(25 \%)$. Galactomannan antigen was positively detected in $(9.4 \%)$ of serum samples, $(22 \%)$ of tracheobronchial aspirates, and in $(22.2 \%)$ bronchoalveoalar lavage fluid samples. The sensitivity (52\%) and specificity (48\%) of culture were much lower than the sensitivity and specificity of galactomannan detection (92\%) and (85\%) respectively. proven IA patient category represented $(2.4 \%)$, while Probable IA represented $(22.4 \%)$, and possible IA represented $(22.2 \%)$. invasive aspergillosis could be a cause of exacerbation in patients with chronic chest diseases and a cause of their unresponsiveness to relevant treatment. Galactomannan antigen detection is a rapid sensitive diagnostic test of IA in those patients that helps the clinicians to rapidly diagnose IA and start antifungal therapy.

\section{Introduction}

Aspergillus spp. exploits many mechanisms that allow their existence as saprophytes \& convey virulence factors to be true human pathogens. Aspergillus fumigatus possess the capability of invading the lung parenchyma, thus it is more likely to be a causative agent of the serious condition known as invasive pulmonary Aspergillosis (Warnock, 2007).

The incidence of invasive fungal infections (IFIs) has increased considerably in recent years because of the increasing population with HIV infection, transplant recipients, cancer patients, and other individuals with 
immune-suppressed status (Hoenigl et al., 2014). Being an abundant component of the Inhaled air, Aspergillus spp. represents one of the leading causes of IFI-related morbidity and mortality (Sherif and Segal, 2010).

This serious infection was primarily described in severely immunocompromised patients such as those with prolonged neutropenia, hematological malignancy, and hematopoietic stem cell and solid organ transplantation recipients. However, there are more and more reports of IPA in patients without the classic risk factors. These reports include patients with chronic obstructive pulmonary disease (COPD) (Vandewoude et al., 2006).

Patients with moderate levels of immune suppression may exhibit different forms of invasive aspergillosis as in those having a "pre- existing structural lung disease" e.g tuberculosis, lung abscess or chronic obstructive lung disease (Ascioglu et al., 2002)

It is not clear why COPD patients may develop IA, An important risk factor is chronic corticosteroid therapy, usually for the treatment of underlying COPD. Another potential risk factor for IA in COPD patients is the severe underlying lung disease however several patients had low FEV1 or described to have stage III or IV COPD. It has been documented that chronic lung diseases predisposes to colonization of airways by Aspergillus spp. and leads to other forms of Aspergillus-related pulmonary disease such as aspergilloma and chronic semi-IA. It is possible that under certain circumstances, such as corticosteroid therapy, acute illness, antibiotics use or comorbid illnesses, this colonization changes to an invasive disease (Hoenigl et al., 2014). Despite the availability of new antifungal drugs, the crude mortality of invasive aspergillosis s 80 to $90 \%$ in the absence of adequate treatment, rapid diagnosis and early start of antifungal therapy are key factors in the successful treatment of IA, as delayed antifungal therapy has a negative impact on the survival of these patients (Hoenigl et al., 2011).

Although conventional diagnostic tests such as microscopy, and culture remain the cornerstone of proving the presence or absence of fungal disease, their yield is low and, therefore, their impact on clinical decisions to treat patients is limited Furthermore, cultures become positive at a late stage of infection and delayed therapy is associated with a poor outcome (Maschmeyer et al., 2009).

Among the most promising diagnostic techniques is the detection of fungal antigens. Galactomannan is a cell wall polysaccharide released by Aspergillus spp. during fungal growth in tissue. A commercially available sandwich enzyme-linked immunosorbent assay (ELISA) detects galactomannan by use of a rat monoclonal antibody (Pasqualotto and Denning, 2005).

Although antigen detection has shown variable performance in large clinical studies revealing both benefits and limitations, the availability of standardized protocols and quality assurance provided by commercial kits have allowed their inclusion in the diagnostic criteria for IFD established by the European Organization for Research and Therapy of Cancer and Mycoses Study Group(EORTC/ MSG) Consensus Group (Basková and Buchta, 2012).

This study aimed at detection of invasive pulmonary aspergellosis in patients with severe chronic chest diseases such as COPD, chronic lung abcess, bronchiectasis, chronic bronchial asthma as a cause of their deteriorated clinical condition and unresponsiveness to relevant treatment, to the 
best of our knowledge, there are no studies available on the detection of invasive pulmonary aspergillosis in patients with chronic chest diseases other than COPD. In order to diagnose this condition, we compared the performance of glactomannan antigen detection in serum of these patients as a rapid, sensitive, and specific diagnostictool to the classic mycological methods.

\section{Patients and Methods}

The study was cross-sectional study, conducted in the period from April 2018 to April 2019 at the microbiology and immunology department and the department of chest diseases, faculty of medicine Sohag University on 85 patients admitted to the department of chest diseases and chest ICU and suspected to have invasive aspergillosis on top of chronic chest diseases such as COPD, chronic bronchial asthma, old pulmonary $\mathrm{TB}$, chronic lung abscess and bronchiectasis according to the revised guidelines of the European Organization for Research and Treatment of Cancer/Invasive Fungal Infections Cooperative Group and the National Institute of Allergy and Infectious Diseases Mycoses Study Group (EORTC/MSG) (De pauw et al., 2008).

Definition of patient groups according to the EORTC/MSG:

* Proven IFA: Isolation of aspergillus species from tissue specimens and blood cultures.

*Probable IFA: Cases of probable IPA require the presence of a host factor, clinical features, and mycological evidence as follows:

Host factor such as:

Recent history of neutropenia $<0.5 \times$ $10^{9}$ neutrophils/L (<500 neutrophils $\left./ \mathrm{mm}^{3}\right)$ for $>10$ days.

Prolonged use of corticosteroids (excluded among patients with allergic bronchopulmonary aspergillosis) at a mean minimum dose of $0.3 \mathrm{mg} / \mathrm{kg} / \mathrm{day}$ of prednisone equivalent for $>3$ weeks

Clinical criteria such:

The presence of 1 of the following 3 signs on CT:Dense, well-circumscribed, lesions(s) with or without a halo sign, Air-crescent sign, and Cavity

Tracheobronchial ulceration, nodule, pseudomembrane, plaque, or eschar seen on bronchoscopic examination.

Mycological criteria such as:

Direct test: direct microscopy and culture, Mold in sputum, bronchoalveolar lavage fluid, or bronchial brush, indicated by Presence of fungal elements of Aspergillus spp. Or Recovery of Aspergillus spp. By culture.

Indirect tests (detection of antigen or cell-wall constituents of Aspergillus as Galactomannan antigen detected in plasma, serum, or bronchoalveolar lavage fluid.

* Possible IFA: Cases of possible IPA require the presence of a host factor and clinical features, but without mycological evidence.

\section{Inclusion criteria}

Patients suspected to have invasive aspergellosis on top of chronic chest disease such as COPD, chronic bronchial asthma, old pulmonary $\mathrm{TB}$, chronic lung abscess and bronchiectasis according to the definitions of EORTC/MSG.

\section{Exclusion criteria}

Active Pulmonary tuberculosis.

Chronic pneumonia.

Renal failure

Hematological malignancy.

Organ transplantation. 
Administration of anti-fungal prophylaxis or empiric therapy with antifungal agents.

All patients were subjected to the following: Full history taking, thorough clinical examination, chest-X-ray, chest CT, and arterial blood gases, ECG, routine laboratory investigations.

\section{Samples}

Tracheal aspirate, bronchial wash fluid and blood samples for culture and isolation of Aspergillus spp.

Serum samples and BAL fluid for detection of circulating galactomannan antigen, measurement of its level.

\section{Culture of collected specimens}

Blood culture was done on conventional blood culture bottles. Subculture was done on SDA with chloramphenicol at $25^{\circ} \mathrm{C}$ and Culture plates were incubated for atleast 4 weeks. Plates were evaluated daily for the first seven days and at least twice per week thereafter. Tracheal aspirates and BAL fluid samples were inoculated directly on SDA with chloramphenicol and cycloheximide and incubated for the same duration.

The isolated Aspergillus spp. Filamentous fungi were identified both macroscopically and microscopically by morphology, color, septation of hyphae pigmentation, pattern of branching, and conidia.

Mould spore suspensions were prepared from 7 -day cultures grown on SDA plates and adjusted spectrophotometrically at a 530-nm wave length to optical densities that ranged from 0.09 to 0.3. Mueller- Hinton agar (Mast group, United Kingdom) was used to perform antifungal susceptibility testing of isolated moulds by disc diffusion method.
The entire surfaces of MHA agar plates (150 $\mathrm{mm}$ ) were inoculated simultaneously in three directions with a nontoxic cotton swab dipped in the mould spore suspensions. The inoculated agar was allowed to dry for 15 to 30 min. The antifungal discs (clotrimazole, itraconazole, voriconazole, Ketoconazole, and amphotericine B) were applied using with centers at least $24 \mathrm{~mm}$ apart. Plates were incubated at $25 \pm 2^{\circ} \mathrm{C}$. Zone diameters in the disk diffusion assay were measured to the nearest whole millimeter at the point where there was a prominent reduction of growth (80\%) after 48to $72 \mathrm{~h}$. Microcolonies inside the zone of inhibition, hyphal filaments bending over the inhibition zones and slight trailing around the edges were ignored when testing azole antifungals but not for polyene antifungals.

Breakpoints are not available for any antifungal agent versus moulds. However; tentative zone diameter categories were assigned as described in table 1 (Sabatelli et al., 2006, Espinel-Ingroff et al., 2007 and Elizabeth, 2008) (Fig. 1).

\section{Serological testing}

Galactomannan antigen was measured in respiratory samples (tracheal aspirates and bronchial wash) and serum of the patients and in control group of normal healthy individuals with comparable age and sex using a commercial sandwich immunoassay Platelia Asprigellus Ag (BioRad, Marnes La Coquette, and France).

Serum samples and bronchial wash fluid were centrifuged at the speed of 2000-3000 rpm for 20 minutes and the supernatant was removed and stored at $-20^{\circ} \mathrm{C}$. the wash buffer $(30 \mathrm{x})$ was diluted in deionized water to prepare 600 $\mathrm{ml}$ of the wash buffer $(1 \mathrm{x})$. The standard was diluted with the standard dilution solution according to the manufacturer instructions 
and $50 \mu \mathrm{l}$ of it was added in the standard well. The sample diluted 5 folds with the sample dilution solution and added to the sample well with touching the well wall and the plate was incubated at $37 \mathrm{C}^{\circ}$ for 30 minutes. The wash buffer was pipetted in each well and still for 30 seconds the aspirated and the wash step was repeated for 5 times. The HRP-conjugate reagent was added $50 \mu 1$ to each well except for the blank well. The incubation and washing steps were repeated. The chromogen solution A and B were added $50 \mu$ l to each well with incubation in the dark for 15 minutes at $37 \mathrm{C}^{\mathrm{o}}$. Stop solution was added to each well and the absorbance was read at 450 $\mathrm{nm}$ taking the blank well as zero value. The actual concentration of galactomannan antigen was calculated by multiplying the sample concentration by the dilution factor.

\section{Statistical analysis}

Quantitative variables were compared using Mann- Whitney test and categorical data with chi-square/ Fisher's exact test. $p$ values of $\leq$ $5 \%$ were considered statistically significant.

\section{Ethical considerations}

Informed oral consent was collected from all patients included in the study, and the study was approved by the institutional committee of research ethics.

\section{Results and Discussion}

A total of 85 patients were enrolled in the study (32 females and 53 males). Their age ranged from 32 to 69 years with a mean age of $(55.8 \pm 9.9)$ with the highest age group lying in the age $\geq 50$ years old. History of smoking was found in 52 patients $(61.1 \%)$ and $(75.3 \%)$ of patients were under corticosteroid therapy (Table 2). Patients included in the study were clinically and radiologically diagnosed as follows: COPD (40) patients, chronic uncontrolled asthma (19) patients, old pulmonary TB (9) patients, bronchiectasis (12) patients, and chronic lung abscess (5) patients (Table 3).

Blood samples for isolation of Aspergillus spp. were collected from all patients included in the study (100\%), while tracheobronchial aspirates were collected from 49 patients (57.6\%), and bronchoalveolar lavage fluid (BAL) were collected from 36 (42.4\%) patients. Aspergillus spp. was isolated from 7 $(14.3 \%)$ tracheobroncheal aspirate samples, 4 (11\%) BAL samples and 2 (2.4\%) blood samples. Aspergillus fumigatus represented (70\%) of isolates while Aspergillus niger represented $(25 \%)$ and small percentage caused Aspergillus terreus (5\%)

Using PlateliaTM Asprigellus Ag detection ELISA kit, galactomannan antigen was positively detected in 8 serum samples (9.4\%), 11 tracheobronchial aspirate samples (22\%), and in 8 (22.2) bronchoalveoalar lavage fluid samples. Comparing the results of different methods used for detection of aspergillus species in various samples collected from patients with chronic lung diseases, 2 cases $(2.4 \%)$ were positive by blood culture, 7 cases $(14.3 \%)$ were positive by culture of tracheobronchial aspirates and 4 cases $(11.1 \%)$ were positive by culture of BAL fluid samples. This number increased to $8(9.4 \%), 11(22.4 \%)$ and $8(22.4 \%)$ by the use of galactomannan $\mathrm{Ag}$ detection test respectively. The sensitivity and specificity of culture were (52\%) and (48\%) which is much lower than the sensitivity and specificity of galactomannan detection $(92 \%)$ and $(85 \%)$ (Table 4). Of the 170 specimens, 13 (7.6\%) yielded a positive culture of Aspergillus spp. From which $2(1.17 \%)$ blood samples yielded a positive culture giving a diagnosis of proven invasive aspergillosis, representing $2.4 \%$ of patients. $11(6.5 \%)$ tracheobronchial aspirates and BAL samples yielded a positive culture 
giving a diagnosis of probable invasive apergillosis with a mycological chriterion, This number increased to $27(15.8 \%)$ specimes which were positive for galactomannan antigen detection by ELISA testing from the total number of samples, expanding the group of probable invasive aspergillosis to $15.8 \%$ which represents $22.4 \%$ of patients. Mycological and clinical chriteria were absent in $74(87.1 \%)$ of patients, from which 17 (20\%) have host factors for invasive aspergillosis according to EORTC/MSG, giving chriteria of diagnosis of possible invasive aspergillosis (Table 4).

The results of antifungal susceptibility testing of the isolated Aspergillus spp. were as follow: the highest susceptibility was to voriconazole 12 (92.3\%), followed by itraconazole 9 (69.2\%), amphotericine B and ketoconazole were equally effective as 7 $(53.8 \%)$ of isolates were susceptible. The least susceptibility was to fluconazole only 1 (7.6\%) was susceptible.

Invasive fungal infections constitute a serious threat to an ever-growing population of immunocompromised individuals and other individuals at risk. Chronic obstructive pulmonary disease (COPD) hasbeen recognized in recent years as an emerging predisposing condition to invasive aspergillosis (IA), frequently in association with mechanical ventilation(MV) and therapy with corticosteroids (Ader, 2010), but little is known about the presence of invasive aspergillosis in other chronic lung diseases especially those with frequent exacerbations or un-controlled on the relevant therapy. Traditional diagnostic methods, such as histopathology and culture, which are still considered the gold standards, have low sensitivity, time consuming which results in delayed initiation of antifungal treatment that underscores the need for the development of new means of detecting fungal infectious agents like newer imaging techniques and non-culture methods, including antigen-based assays, metabolite detection and molecular detection of fungal DNA from body fluid samples(Arvanitis et al., 2014).

In the present cross sectional study we supposed that invasive aspergillosis could be a caused of deteriorated condition of patients with chronic lung disease and their unresponsiveness to therapy especially those with certain risk factors. We collected sputum and blood samples from those patients for isolation of aspergilllus spp. and bronchoalveolar wash fluid and serum samples for assay of galactomannan antigen.

In the present study, most cases of invasive fungal infection was in the age $>50$ years. This is inconsistent with the work of Meersseman (2004) and Montagna et al., (2013) where most of cases tend to occur in high age (older than 60 years), and this can be attributed to the increased incidence of invasive mycoses with bipolarity of age in association with diminished immunity. On the contrary, Soliman et al., (2012) detected most of invasive fungal infection cases within younger age group, as this study was conducted on patient with hematological malignancy which show higher incidence in this group of age.

As regarding host factors distribution, it was found that $75.3 \%$ of the studied patients were under corticosteroid therapy, $15.3 \%$ were neutropenic. This is in agreement with Meersseman et al., (2004) who found that the highest percentage of studied patients was undercorticosteroid therapy, followed by patients who were neutropenic. Similarly, in the study of Slavin et al., (2010) the highest percentage of studied patients were under corticosteroid therapy while cases that were on immune-suppressive therapy and neutropenic cases nearly were same 
percentage. High incidence of IA with corticosteroids is due to suppression of the immune system specially the cellular component (Sipsas and Kontoyiannis, 2012). Meersseman et al., (2004) explained that Corticosteroids substantially impair macrophage killing of Aspergillus spores and mononuclear cell killing of Aspergillus hyphae, also threshold steroid level varies according to the type of patients, and emphasized that in the setting of underlying lung disease there is a risk factor for IA at much lower doses.

Distribution of risk factors among the studied patients with invasive aspergillosis with mycological and clinical criteria revealed a high association of IA with corticosteroid therapy ( $p$ value 0.001),neutropenia( $p$ value 0.01 ), and diabetes ( $p$ value 0.04). This result is consistent with Slavin et al., (2010)who found that the highest percent of studied patients had diabetes. Also, in the study of Montagna et al., (2013), there was an association between steroid therapy, diabetes, and neutropenia from one side and presence of invasive aspergillosis from the other side.

Aspergillus spp. was isolated from (7.6\%) of samples, of which A. fumigatus was responsible for $(70 \%), A$. niger was responsible for (25\%), and A. terreus (5\%). These findings were consistent with (Ulrike and Lass-Flörl,2011) who reported that the most common species causing aspergillosis is A. fumigatus, also Jane et al., (2011) who found that A.fumigatus causes $44 \%$ of invasive aspergillosis cases. A. fumigatusis more thermotolerant than other diseasecausing species, growing well at $37^{\circ} \mathrm{C}$, and several studies suggested that the radial growth and germination rate of aspergilli at $37^{\circ}$ Ccorrelate with pathogenicity. Moreover, being resistant to oxidative stress; A.fumigatus detoxifies oxidative threats viaglutathione synthesis and oxidoreductase activity (Dagenais and Keller, 2009). Furthermore, Ulrike and Lass-Flörl (2011) found that the most common species causing aspergillosis is A. fumigatus, accounting for approximately $90 \%$ of aspergillus infections.

In order to overcome the limitations of conventional diagnostic tests, several studies have focused on the development of nonculture based methods with superior sensitivity and quicker turnaround time, such as the detection of fungal components or metabolites, as an alternative approach for the early detection and identification of the causative fungal species (Yeo and Wong, 2002).

Mycological culture of BAL fluid is indispensable, as antifungal susceptibility testing is currently available from culture only. Culture may also grow other molds, like Mucorales, which do not result in positive GM or BDG levels. It has to be emphasized, however, that the low sensitivity of conventional culture is its major drawback. Lass-Flörl and colleagues (Lass-Flörl et al., 2007) have reported a sensitivity as low as $34 \%$ of conventional mycological culture (21/61 positive samples).

On the other hand a study conducted by (Nguyenet al., 2007), tested for the presence of Aspergillus GM antigen in nonimmunocompromised patients, including those with COPD in BAL. They found that BAL GM testing was no more sensitive than the combination of BAL microscopy and culture and exhibited a lower PPV than these tests, and it only increased the likelihood of obtaining false-positive results. We thus recommend that GM detection be done in serum and BAL in combination with BAL culture for Aspergillus.

In this study, aspergillus spp. was isolated from a total of 13 samples (15.3\%), while 
galactomannan antigen was detected in 27 samples $(31.7 \%)$. the sensitivity of mycological culture from various samples was found to be around (52\%) compared to galactomannan detection $(92 \%)$, but it allowed the advantage of determining the antigungal susceptibility of the isolates. Galactomannan detection in the same samples was more sensitive and considered a rapid diagnostic tool in diagnosis of invasive aspergillosis in suspected cases.

Invasive Aspergillosis in our study were defined using EORTC/MSG Consensus Group criteria (De Pauw et al., 2008), and according to these criteria we found that $1.17 \%$ of cases were classified as proven IA through isolation of aspergillus spp. from blood samples, $15.8 \%$ were probable IA through the presence of clinical and mycological (galactomannan detection) chriteria and $87.1 \%$ were possible invasive fungal infection through the presence of host risk factor only

In a study by (Graf et al., 2011), Probable IPA was diagnosed in $24 \%$ of the patients, using either culture or antigen detection as a microbiological indicator. They included 704 patients in their study, which probably makes their results more representative. However, they included patients with severe underlying diseases and some transplant patients, thus their study encompasses a wider range of risk factors (Table 4).

Table.1 Tentative zone diameter categories of antifungal agents against isolated filamentous fungi

\begin{tabular}{|c|c|c|c|c|}
\hline Antifungal & Disc content & \multicolumn{3}{|c|}{ Zone diameter in $\mathbf{m m}$} \\
\cline { 2 - 4 } & & S & I & R \\
\hline Amphotericine B & $100 \mathrm{U}$ & $\geq 15$ & $13-14$ & $\leq \mathbf{1 2}$ \\
\hline Fluconazole & $10 \mu \mathrm{g}$ & $\geq 17$ & $14-16$ & $\leq \mathbf{1 3}$ \\
\hline Itraconazole & $10 \mu \mathrm{g}$ & $\geq 17$ & $14-16$ & $\leq \mathbf{1 3}$ \\
\hline Voriconazole & $1 \mu \mathrm{g}$ & $\geq 17$ & $14-16$ & $\leq \mathbf{1 3}$ \\
\hline Ketoconazole & $10 \mu \mathrm{g}$ & $\geq 28$ & $27-21$ & $\leq \mathbf{2 0}$ \\
\hline Clotrimazole & $\mathbf{1 0} \boldsymbol{\mu g}$ & $\geq \mathbf{2 0}$ & $\mathbf{1 2}-\mathbf{1 9}$ & $\leq \mathbf{1 1}$ \\
\hline
\end{tabular}

Table. 2 distribution of risk factors among patients with invasive aspergillosis

\begin{tabular}{|c|c|}
\hline Variable & Percentage \\
\hline Age & Mean age $(59.8 \pm 9.9)$ \\
\hline $\begin{array}{c}\text { Sex (males) } \\
\text { Corticosteroid } \\
\text { therapy }\end{array}$ & $53(62.35 \%)$ \\
\hline neutropenia & $64(75.3 \%)$ \\
\hline History of smoking & $13(15.3 \%)$ \\
\hline Diabetes & $52(61.17 \%)$ \\
\hline Anemia & $22(85.8 \%)$ \\
\hline Hypertension & $39(45.8 \%)$ \\
\hline Cardiac disease & $15(17.6 \%)$ \\
\hline
\end{tabular}


Table.3 Distribution of study groups according to disease category

\begin{tabular}{|c|c|}
\hline \multicolumn{2}{|c|}{ Classification of patients according to disease category } \\
\hline Disease category & Percentages of patients \\
\hline COPD & $40(47 \%)$ \\
Stage III & $28(32.9 \%)$ \\
Stage IV & $12(14.1 \%)$ \\
\hline $\begin{array}{c}\text { Chronic uncontrolled } \\
\text { asthma }\end{array}$ & $19(22.3 \%)$ \\
\hline Bronchiectasis & $12(14.3 \%)$ \\
\hline Old pulmonary TB & $9(10.6 \%)$ \\
\hline Chronic lung abscess & $5(5.8 \%)$ \\
\hline Average pulmonary functions of the study group \\
\hline $\begin{array}{c}\text { Forced vital capacity (FVC) } \\
\text { Forced expiratory volume } \\
\text { in 1 sec (FEV1) }\end{array}$ & $72 \%$ \\
\hline FEV1/FVC & $60 \%$ \\
\hline
\end{tabular}

Table.4 the comparative results of culture and galactomannan detection in different samples

\begin{tabular}{|c|c|c|c|}
\hline Type of sample & $\begin{array}{c}\text { Number of } \\
\text { samples }\end{array}$ & \multicolumn{2}{|c|}{ Method used } \\
\cline { 3 - 4 } & & Culture & $\begin{array}{c}\text { Galactomannan } \\
\text { Ag detection }\end{array}$ \\
\hline Blood and serum samples & $\mathbf{8 5 ( 1 0 0 \% )}$ & $\mathbf{2 ( 2 . 4 \% )}$ & $\mathbf{8 ( 9 . 4 \% )}$ \\
\hline $\begin{array}{c}\text { Tracheobronchial aspirates } \\
\text { Bronchoalveolar lavage } \\
\text { fluid }\end{array}$ & $\mathbf{4 9 ( 5 7 . 6 \% )}$ & $\mathbf{7 ( 1 4 . 3 \% )}$ & $\mathbf{1 1 ( 2 2 . 4 \% )}$ \\
\hline \multicolumn{2}{|c|}{ Total= 85 samples (100\%) } \\
\hline
\end{tabular}

Table.5 Classification of patient groups according to EORTC/MSG criteria

\begin{tabular}{|c|c|c|c|}
\hline & \multirow[t]{2}{*}{ Criteria for classification } & \multicolumn{2}{|c|}{$\begin{array}{l}\text { Number and } \\
\text { Percentage of } \\
\text { patients }\end{array}$} \\
\hline & & NO. & $\%$ \\
\hline Proven IA & Isolation of aspergillus species from blood & 2 & $2.4 \%$ \\
\hline Probable IA & $\begin{array}{l}\text { Isolation of aspergillus spp. From BAL fluid and } \\
\text { tracheobronchial aspirates } \\
\text { Detection of positive cut off value for galactomannan } \\
\mathrm{Ag} \text { in specimens of patients }\end{array}$ & 19 & $22.4 \%$ \\
\hline Possible IA & $\begin{array}{l}\text { Presence of a host factor with absence of clinical and } \\
\text { mycological criteria for diagnosis. }\end{array}$ & 17 & $20.0 \%$ \\
\hline
\end{tabular}


Fig.1 a) Macroscopic appearance of Aspergillus fumigatus. b) Macroscopic appearance of Aspergillus terreus.c) Microscopic appearance of Aspergillus terreus

a)

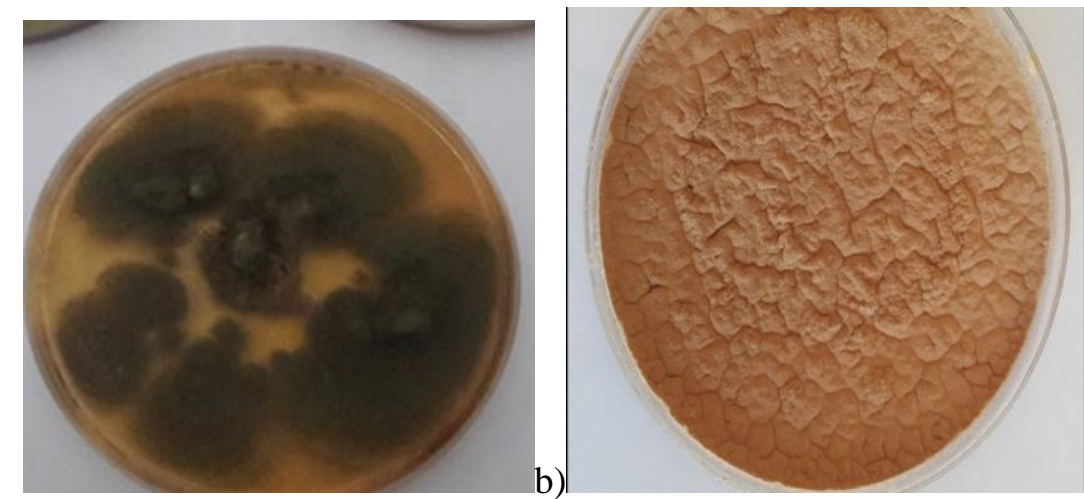

c)

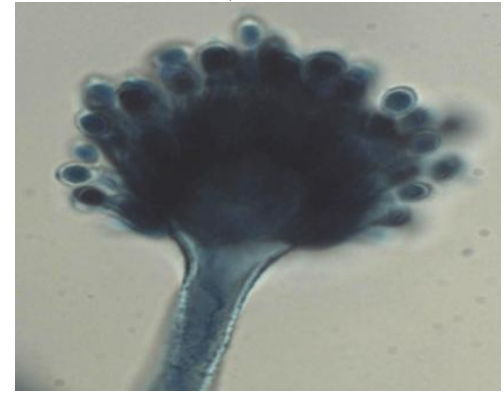

Recent studies suggest that IA may be frequent inpatients admitted to the ICU (Ader et al., 2010) and (Guinea et al., 2016) such as COPD patients who seem to be particularly at risk, which has been associated with previous airway colonization with Aspergillus species, in a context of severe structural lung disease, requirement of invasive MV and therapy with corticosteroids Based on these observations, we selected for inclusion in our study patients and we included also patients with chronic chest diseases other than COPD who were theoretically at risk for IA.

Even though the small frequency of probable IA in our study, most of the other studies in COPD patients showed similar/lower rates of IA. Gao et al., (2010) studied 261 patients with exacerbated COPD and found that only $1.9 \%$ had IA. In the study by (Guinea et al., 2016), 53 cases of IA were found among 14 thousands COPD patients requiring hospital admission (incidence 0.4\%). On the other extreme, Belgian studies showed IA frequencies as high as 6-24\% in ICU patients (Meersseman et al., 2008) with some of these individuals also being diagnosed with COPD (7.7-31.4\%).

The conclusion of this study is that invasive aspergillosis could be a cause of exacerbation in patients with chronic chest diseases that need ICU admission and could be a cause of their unresponsiveness to relevant treatment. Clinicians should be aware of and familiar with the newer diagnostic modalities of IA such as galactomannan antigen detection in conjunction with older diagnostic techniques, to rapidly diagnose IA and start proper antifungal therapy.

\section{References}

Ader, F. 2010.Invasive pulmonary aspergillosis in patients with chronic obstructive pulmonary disease: an emerging fungal disease. Curr Infect Dis Rep., 12:409-16.

Arvanitis, M., Anagnostou, T., Fuchs, B. B., 
Caliendo, A.M., Mylonakis, E. 2014. Molecular and nonmolecular diagnostic methods for invasive fungal infections. Clin.Microbiol. Rev., 27(3): 490.

Ascioglu, S., Rex,JH., de Pauw, B., Bennett. JE., Bille, J., Crokaert, F., Denning, DW., Donnelly, JP., Edwards, JE., Erjavec, Z., Fiere, D., Lortholary, O., Maertens, J., Meis, JF., Patterson, TF., Ritter, J., Selleslag, D., Shah, PM., Stevens, DA., and Walsh, TJ. 2002. Defining Opportunistic Invasive Fungal Infections in Immunocompromised Patients with Cancer and Hematopoietic Stem Cell Transplants: An International Consensus. Clinical Infectious Diseases. 34:7-14.

Ba ková, L., Buchta, V. 2012. Laboratory diagnostics of invasive fungal infections: an overview with emphasis on molecular approach. Folia Microbiol., 57: 421- 430.

Dagenais, T.R., Keller, N.P. 2009.Pathogenesis of Aspergillus fumigatus in invasive aspergillosis. Clin. Microbiol. Rev., 22(3): 447465.

De Pauw, B., Walsh, T.J., Donnelly, J.P., Stevens, D.A., Edwards, J.E., Calandra, T., Pappas, P.G., Maertens, J., Lortholary, O., Kauffman, C.A., Denning, D.W., Patterson, T.F., Maschmeyer, G., Bille, J., Dismukes, W.E., Herbrecht, R., Hope, W.W., Kibbler, C.C., Kullberg, B.J., Marr, K.A., $\mathrm{Mu}$ oz, P., Odds, F.C., Perfect, J.R., Restrepo, A., Ruhnke, M., Segal, B.H., Sobel, J.D., Sorrell, T.C., Viscoli, C., Wingard, J.R., Zaoutis, T., Bennett, 2008.Revised definitions of invasive fungal disease from the European Organization for Research and Treatment of Cancer/Invasive Fungal Infections Cooperative Group and the National Institute of Allergy and Infectious Diseases Mycoses Study Group consensus group (EORTC/MSG) Infect. Dis., 46: 18131821.

Guinea, J., Torres-Narbona, M., Gijo'n, P., Muño, P., Pozo, F., Pela'ez, T., de Miguel, J., Bouza, E. 2016.Pulmonary aspergillosis in patients with chronic obstructive pulmonary disease: incidence, risk factors, and outcome. Clin Microbiol Infect., 16:870-7.

Hoenigl,M., Prattes, J., Spiess, B., Wagner, J., Prueller, F., Raggam, B. 2014. Performance of Galactomannan, Beta-DGlucan, Aspergillus Lateral Flow Device, conventional Culture, and PCR Tests with Bronchoalveolar Lavage Fluid for Diagnosis of Invasive Pulmonary Aspergillosis. Journal of Clinical Microbiology. 52 (6): 2039-2045.

Hoenigl, M., Zollner-Schwetz, I., Sill, H., Linkesch, W., Lass-Flörl, C., Schnedl, WJ., Krause, R. 2011.Epidemiology of invasive fungal infections and rationale for antifungal therapy in patients with haematological malignancies. Mycoses., 54:454-459.

Jane, K., James, I., Sanjeet, S. 2011.Epidemiology and treatment approaches in management of invasive fungal infections. Clin. Epidemiol., 3: 175 191.

Lass-Flörl, C., Resch, G., Nachbaur, D., Mayr, A., Gastl, G., Auberger, J., Bialek, R., Freund, MC. 2007. The value of computed tomography-guided percutaneous lung biopsy for diagnosis of invasive fungal infection in immunocompromised patients. Clin. Infect. Dis., 45:101-104.

Maschmeyer, G., Beinert, T., Buchheidt, D., Cornely, O.A., Einsele, H., Heinz, W., Heussel, C.P., Kahl, C., Kiehl, M., Lorenz, J., Hof, H., Mattiuzzi, M. 2009. Diagnosis and antimicrobial therapy of lung infiltrates in febrile neutropenic patients: Guidelines of the infectious diseases working party of the German Society of Haematology and Oncology. Eur. J. Cancer, 45: 24622472.

Meersseman, W., Lagrou, K., Maertens. J., Wilmer, A., Hermans, G., Vanderschueren, S., Spriet, I., Verbeken, E., Van Wijngaerden, E. 2008. Galactomannan in bronchoalveolar lavage fluid: a tool for diagnosing aspergillosis in intensive care unitpatients. Am J Respir Crit Care Med., 177: 27-34. 
Meersseman, W., Vandecasteele, S.J., Wilmer, A., Verbeken, E., Peetermans, W.E., Wijngaerden, E.V. 2004. Invasive aspergillosis in critically ill patients without malignancy. Am. J. Respir. Crit. Care Med., 170: 621625.

Montagna, M.T., Caggiano, G., Lovero, O., De Giglio, O., Coretti, C., Cuna, T., Iatta, R., Giglio, M., Dalfino, L., Bruno, F., Puntillo, F. 2013. Epidemiology of invasive fungal infections in the intensive care unit: results of a multicenter Italian survey (AURORA Project). Infection., 41: 645653

Nguyen, MH., Jaber, R., Leather, HL., Wingard, JR., Staley, B., Wheat, LJ., Cline, CL., Baz, M., and Clancy, CJ. 2007. Use of bronchoalveolar lavage to detect galactomannan for diagnosis of pulmonary aspergillosis among nonimmunocompromised hosts. J. Clin. Microbiol., Pp. 2787-2792.

Pasqualotto, D.A., Denning D.W. 2005. Diagnosis of invasive fungal infections Current limitations of classical and new diagnostic methods. Business Briefing : European Oncology Review. Pp. 111.

Sherif, R., and Segal, BH. 2010. Pulmonary Aspergillosis: clinical presentation, diagnostictests, management and complications. Curr. Opin. Pulm Med., 16(3): 242-250. Gaston K J Global pattern in biodiversity. Nature., 405(1):220-7.
Slavin, M.A., Sorrell, T.C., Marriott, D., Thursky, K.A., Quoc Nguyen, Q., Ellis D.H., Morrissey, C.O., Sharon C.A.C. 2010. Candidaemia in adult cancer patients: risks for fluconazole-resistant isolates and death. J. Antimicrob. Chemother., 65:1042-1051.

Soliman, M.H., Azab, M.M., Abu Taleb, F.M., Mohamed, N.A. 2012.Identification of different types of fungi isolated from patients with haematologic malignancy and from their surrounding exogenous sources. Z.U.M.J., 18(5): 951-961.

Ulrike, B., Lass-Flörl, C. 2011. Epidemiology of Invasive Fungal Infections in the Mediterranean Area. Mediterr. J. Hematol. Infect. Dis., 3; Open Journal System.

Vandewoude,KH., Blot, SI., Depuydt, P. 2006. Clinical relevance of aspergillus isolation from respiratory tract samples in critically illpatients. Crit Care. Pp. 10-31.

Warnock, DW. 2007. Fungi in Medical Microbiology; A Guide to microbial infections, pathogenesis, immunity, laboratory diagnosis, and control Greenwood, Slack, R., Peuthrer, J., Barer, M., Seventeenth, ed. 2007. Churchil Livingstone Elsevier. P.612-614.

Yeo, S.F., Wong, B. 2002. Current status of nonculture methods for diagnosis of invasive fungal infections. Clin. Microbiol. Rev., 15: 465-484.

\section{How to cite this article:}

Asmaa Nasr Eldin Thabet and Kamal Abdel Sattar Ata. 2019. Glactomannan Index as a Rapid Diagnostic Tool for Invasive Pulmonary Aspergillosis in Patients with Chronic Lung Diseases. Int.J.Curr.Microbiol.App.Sci. 8(06): 1123-1134. doi: https://doi.org/10.20546/ijcmas.2019.806.139 